\title{
Modeling of a Saturated Switched Reluctance Motor Using an Operating Point Analysis and the Unsaturated Torque Equation
}

\author{
Nicholas J. Nagel, Associate Member, IEEE, and Robert D. Lorenz, Fellow, IEEE
}

\begin{abstract}
This paper describes torque production in a saturated switched reluctance motor (SRM). The paper begins by deriving the equations for torque if the SRM is unsaturated. It then develops an operating point model for torque in a saturated SRM using the unsaturated form of the torque equation. This results in a methodology which makes the control algorithms applicable to either saturated or unsaturated SRM's.
\end{abstract}

Index Terms-Co-energy, saturation modeling, smooth torque, switched reluctance motor.

\section{INTRODUCTION}

$\mathbf{S}$ WITCHED reluctance motors (SRM's) have been the focus of much research over the past three decades. With improvements in power electronics and microprocessors, this trend will continue to grow.

The smooth production of electromagnetic torque is a desirable characteristic of any motor. While smooth torque production has been achieved in dc and ac induction and synchronous machines, it has not, historically, been achieved by SRM's. Smooth torque production is a necessary, but not sufficient, requirement in order to achieve servo grade performance from a motor. Another necessary condition is dynamic torque control. Few researchers have focused on the dynamics of electromagnetic torque production in SRM's.

Typically, research has either focused on simple, practical solutions of control of the SRM or complex mathematical algorithms. Most of these techniques use only one phase at a time to produce the commanded torque. More recently, researchers have used torque sharing (or contour) functions to smoothly transition torque between two or more phases [1]-[7].

All of these techniques require a method for the prediction of torque in each of the phases of the SRM. Most of these techniques are greatly simplified by first assuming an unsaturated SRM. It is the goal of this paper to present a generalized oper-

Paper IPCSD 99-86, presented at the 1999 Industry Applications Society Annual Meeting, Phoenix, AZ, October 3-7, and approved for publication in the IEEE TRANSACTIONS ON INDUSTRY APPLICATIONS by the Electric Machines Committee of the IEEE Industry Applications Society. Manuscript submitted for review June 1, 1999 and released for publication December 10, 1999. This work was supported by the Wisconsin Electric Machines and Power Electronics Consortium, University of Wisconsin, Madison.

N. J. Nagel is with MPC Products Corporation, Skokie, IL 60077 USA (e-mail: nnagel@MPCProducts.com).

R. D. Lorenz is with the Department of Mechanical Engineering and the Department of Electrical and Computer Engineering, University of Wisconsin, Madison, WI 53706 USA (e-mail: lorenz@engr.wisc.edu).

Publisher Item Identifier S 0093-9994(00)03160-1. ating point model for torque in a saturated SRM using the unsaturated form of the torque equation.

\section{TORQUE PRODUCTION IN SRM'S}

In any type of electromechanical device, an energy balance can be used to derive the mechanical work in terms of the input energy, the dissipated energy, and the stored energy. In a reluctance device, which has wire wound (or coiled) around a highly permeable material and a moveable part of highly permeable material, the mechanical work required to move the permeable material is the supplied electrical input energy minus the energy dissipated in the resistance of the coil minus the stored field energy. This is expressed mathematically as given in

$$
d W_{\text {mech }}=d W_{\text {elec }}-d W_{f}
$$

where

$d W_{\text {mech }} \quad$ differential output of mechanical energy;

$d W_{\text {elec }} \quad$ differential input in electrical energy of the coil; $d W_{f} \quad$ differential increase in stored magnetic energy.

For one of the phases of an SRM, the electrical energy of the coil is given in

$$
d W_{\text {elec }}=(v-i r) i d t=e i d t .
$$

Using Faraday's Law of induced voltage in a coil, (2) can be expressed as in

$$
d W_{\mathrm{elec}}=\frac{d \lambda}{d t} i d t=i d \lambda
$$

Using (3) in (1) gives

$$
d W_{\text {mech }}=i d \lambda-d W_{f} .
$$

Noting from the calculus of several variables that, given a function $f(x, y)$, then

$$
d f(x, y)=\frac{\partial f}{\partial x} d x+\frac{\partial f}{\partial y} d y .
$$

Two independent variables are necessary to describe the total energy of the system. These two independent variables are either flux linkage (integrated voltage) and movable armature position or current and movable armature position. In an SRM, the movable armature is the rotor, thus rotor position is always a state variable. The choice of flux linkage or current as the other state variable is arbitrary and each leads to expressions for torque in the SRM, as will be shown in the next two sections. 


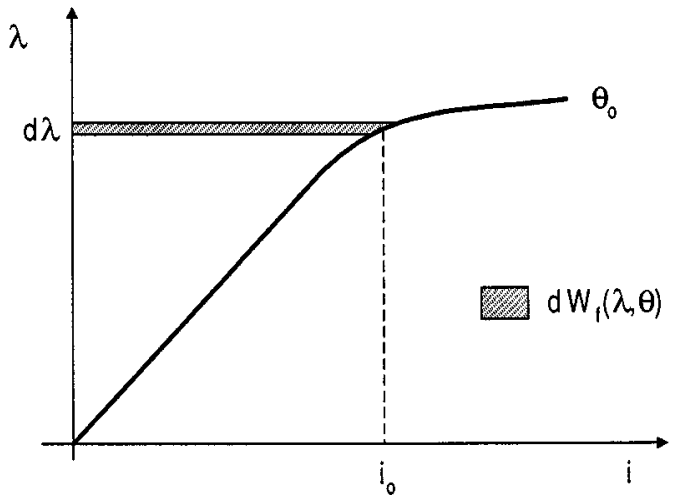

Fig. 1. Plot of flux linkage versus current demonstrating current as a function of differential stored energy with respect to a differential change in flux linkage.

\section{A. Torque Production in an SRM Using Flux Linkage and Rotor Position as State Variables}

The torque and stored field energy in an SRM can be expressed as a function of rotor position and flux linkage as follows. Assuming the current in the machine, as well as the stored magnetic energy and the electrical energy, are functions of flux linkage and position, then the energy balance is given by

$$
d W_{\operatorname{mech}}(\lambda, \theta)=i(\lambda, \theta) d \lambda-d W_{f}(\lambda, \theta) .
$$

The differential field energy can be expressed from (5) as given in

$$
d W_{f}(\lambda, \theta)=\frac{\partial W_{f}(\lambda, \theta)}{\partial \lambda} d \lambda+\frac{\partial W_{f}(\lambda, \theta)}{\partial \theta} d \theta .
$$

Using (7) in (6) and noting that the differential amount of mechanical work is the instantaneous torque times the differential change in position, then (6) can be re-written as shown in

$$
\begin{aligned}
T(\lambda, \theta) d \theta= & \left(i(\lambda, \theta)-\frac{\partial W_{f}(\lambda, \theta)}{\partial \lambda}\right) d \lambda \\
& -\frac{\partial W_{f}(\lambda, \theta)}{\partial \theta} d \theta
\end{aligned}
$$

Equating like coefficients in (8) gives the following two equations:

$$
\begin{gathered}
i(\lambda, \theta)=\left.\frac{\partial W_{f}(\lambda, \theta)}{\partial \lambda}\right|_{\theta=\mathrm{const}} . \\
T(\lambda, \theta)=-\left.\frac{\partial W_{f}(\lambda, \theta)}{\partial \theta}\right|_{\lambda=\mathrm{const}} .
\end{gathered}
$$

Equation (9) is a constraint equation and states that the current is equal to the differential change in stored field energy with respect to a differential change in flux linkage with rotor position held constant. This can be seen most easily from Fig. 1.

The value of current expressed in (9) is a differential, implying that the current is evaluated as the differential flux linkage tends toward zero. Fig. 1 shows that, as the differential flux linkage tends toward zero, the differential stored energy tends toward a line (similar to a delta function) and there is a

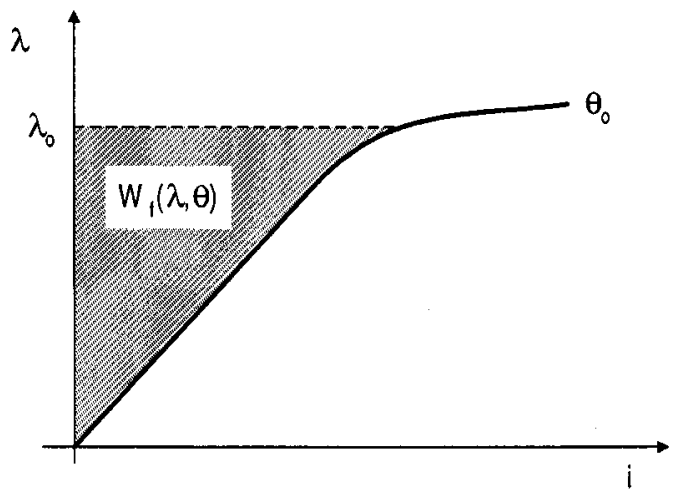

Fig. 2. Plot of flux linkage versus current showing the stored energy in the magnetic field for a given level of flux linkage at a given position.

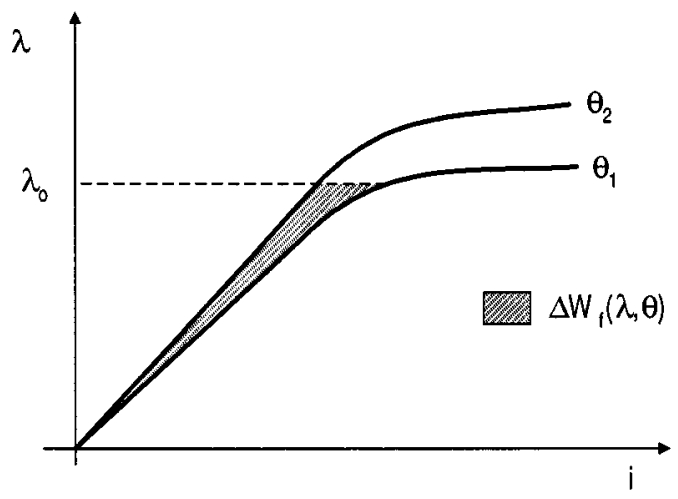

Fig. 3. Plot of flux linkage versus current showing the differential stored energy as a function of position for a fixed flux linkage.

one-to-one mapping between this differential horizontal line of flux linkage and the current.

Alternatively, (9) can be integrated to give an expression for the stored energy in the magnetic field for a given rotor position

$$
W_{f}\left(\lambda_{o}, \theta_{o}\right)=\int_{0}^{\lambda_{o}} i\left(\lambda, \theta_{o}\right) d \lambda
$$

This is shown graphically in Fig. 2 as the area bounded by the flux-linkage axis and the curve of flux linkage versus current.

Equation (10) gives the desired expression for instantaneous torque in terms of the differential change in stored energy with respect to position, evaluated at a constant flux linkage. Fig. 3 shows the differential stored field energy between two rotor positions as the change in stored field energy with a constant flux linkage.

The average torque is equal to the area enclosed by this change in stored field energy divided by the change in rotor position. The instantaneous torque is the differential area divided by the change in rotor position as the change in rotor positions tends toward zero.

\section{B. Torque Production in an SRM Using Phase Current and Rotor Position as State Variables}

In the previous section, the torque and stored field energy in an SRM were expressed as functions of rotor position and flux linkage. The independent variable, flux linkage, can be replaced by current as follows. Assuming the flux linkage in the machine, 


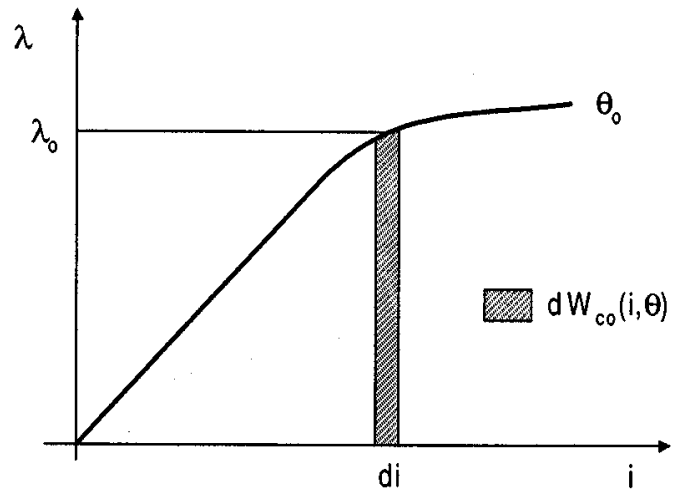

Fig. 4. Plot of flux linkage versus current demonstrating flux linkage as a function of differential co-energy with respect to a differential change in current.

as well as the stored magnetic energy and the electrical energy, are functions of current and position, then the energy balance is given in

$$
d W_{\text {mech }}(i, \theta)=i d \lambda(i, \theta)-d W_{f}(i, \theta) .
$$

It is common in reluctance actuator analysis to define a term known as co-energy. This term simplifies the analysis and is defined in

$$
W_{\mathrm{co}}(i, \theta)=i \lambda(i, \theta)-W_{f}(i, \theta) .
$$

Differentiating (13) gives

$$
d W_{\mathrm{co}}(i, \theta)=i d \lambda(i, \theta)+\lambda(i, \theta) d i-d W_{f}(i, \theta) .
$$

Solving (14) for $d W_{f}(i, \theta)$ and substituting into (12) gives

$$
d W_{\text {mech }}(i, \theta)=-\lambda(i, \theta) d i+d W_{\mathrm{co}}(i, \theta) .
$$

From (5), the differential co-energy can be expanded to

$$
d W_{\mathrm{co}}(i, \theta)=\frac{\partial W_{\mathrm{co}}(i, \theta)}{\partial i} d i+\frac{\partial W_{\mathrm{co}}(i, \theta)}{\partial \theta} d \theta .
$$

Again noting that the differential amount of mechanical work is the instantaneous torque times the differential change in position, and substituting (16) into (15) gives

$$
T(i, \theta) d \theta=\left(\lambda(i, \theta)-\frac{\partial W_{\mathrm{co}}(i, \theta)}{\partial i}\right) d i+\frac{\partial W_{\mathrm{co}}(i, \theta)}{\partial \theta} d \theta .
$$

Equating like coefficients in (17) gives the following two equations

$$
\begin{gathered}
\lambda(i, \theta)=\left.\frac{\partial W_{\mathrm{co}}(i, \theta)}{\partial i}\right|_{\theta=\mathrm{const}} \\
T(i, \theta)=\left.\frac{\partial W_{\mathrm{co}}(i, \theta)}{\partial \theta}\right|_{i=\mathrm{const}} .
\end{gathered}
$$

In a similar fashion as above, (18) expresses flux linkage as the differential of co-energy with respect to a differential change in current. As the limit of this differential current tends toward zero, the co-energy tends toward a line. Again, there is

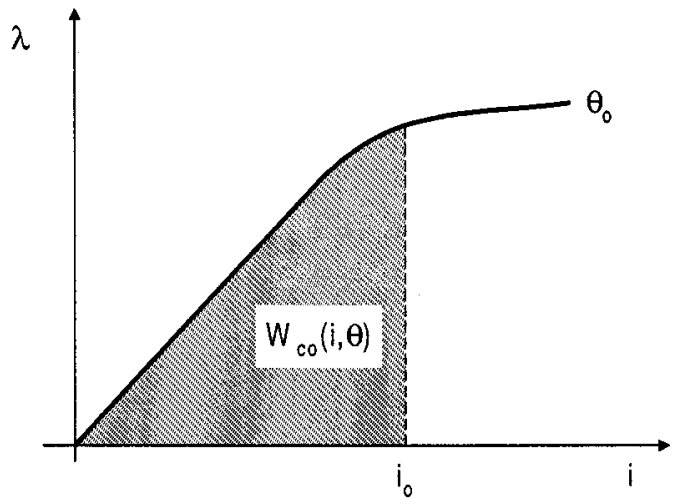

Fig. 5. Plot of flux linkage versus current showing the co-energy for a given current level at a given position.

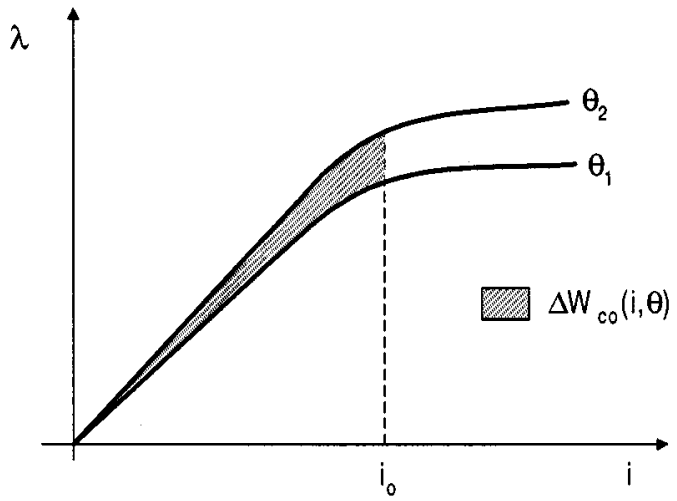

Fig. 6. Plot of flux linkage versus current showing the change in co-energy as a function of position for a fixed current.

a one-to-one mapping between this vertical line and the flux linkage, as shown in Fig. 4.

Alternatively, (18) can be integrated to give an expression for the co-energy for a given rotor position as in

$$
W_{\mathrm{co}}\left(i_{o}, \theta_{o}\right)=\int_{0}^{i_{o}} \lambda\left(i, \theta_{o}\right) d i .
$$

This is shown graphically in Fig. 5 as the area bounded by the current axis and the curve of flux linkage versus current. This follows directly from the definition of co-energy given in (13) as the rectangular area given by $\lambda-i$ minus the stored magnetic field energy.

Equation (19) gives an expression for instantaneous torque in terms of the differential change in co-energy with respect to position, evaluated at a constant current. Fig. 6 shows the change in co-energy as a function of position with a constant current.

The average torque is equal to the area enclosed by this change in co-energy divided by the change in position. The instantaneous torque is the differential co-energy as the change in position tends toward zero.

\section{OPerating Point TORQue Model IN AN SRM Using Flux LinKage and Rotor Position as State Variables}

To begin the operating point analysis of the SRM in terms of flux linkage and rotor position, the expression for torque must first be derived for the linear portion of the flux linkage versus 


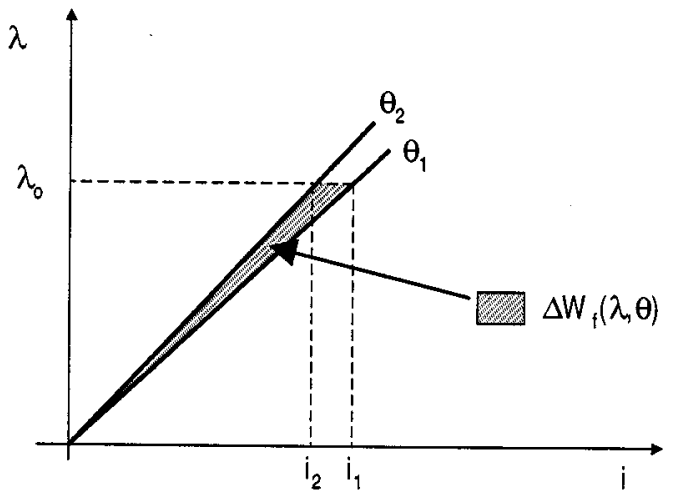

Fig. 7. Plot of flux linkage versus current showing the change in stored field energy as a function of position for a fixed flux linkage.

current curve. Fig. 7 shows the change in stored field energy with a change in rotor position for the linear part of the flux linkage versus current curve. The change in stored field energy is the difference between the triangular areas of the flux linkage versus current curves. The base of both of these triangular areas is the constant flux linkage, shown as $\lambda_{\circ}$ in Fig. 7.

The heights of the two triangles are the corresponding currents, shown as $i_{1}$ and $i_{2}$. The equation for torque for the linear magnetic case for one phase of the SRM in terms of the flux linkage and rotor position is given by

$$
T\left(\lambda_{o}, \theta\right)=-\lim _{\Delta \theta \rightarrow 0}\left(\frac{1}{2} \lambda_{o} \frac{i_{2}\left(\lambda_{o}, \theta\right)-i_{1}\left(\lambda_{o}, \theta\right)}{\Delta \theta}\right) .
$$

The relationship between flux linkage and current with reluctance is given by

$$
i(\lambda, \theta)=\frac{\lambda}{N^{2}} \Re(\lambda, \theta)
$$

where

$$
\Re(\lambda, \theta)=\frac{N^{2}}{L(\lambda, \theta)} .
$$

When the machine is not saturated, the inductance and, therefore, reluctance are no longer functions of flux linkage. They are still, however, functions of rotor position. Using (22) in (21) gives

$$
\begin{aligned}
T\left(\lambda_{o}, \theta\right) & =-\lim _{\Delta \theta \rightarrow 0}\left(\frac{1}{2} \frac{\lambda_{0}^{2}}{N^{2}} \frac{\Delta \Re(\theta)}{\Delta \theta}\right) \\
& =-\left(\frac{1}{2} \frac{\lambda_{0}^{2}}{N^{2}} \frac{d \Re(\theta)}{d \theta}\right) .
\end{aligned}
$$

Having derived the torque in terms of the flux linkage and rotor position for the unsaturated case, an operating point model for torque as a function of flux linkage and rotor position needs to be derived. It is interesting to note that several researchers have stated that the expression for torque (23) was valid for any flux level, not just when the machine was unsaturated [1], [14].

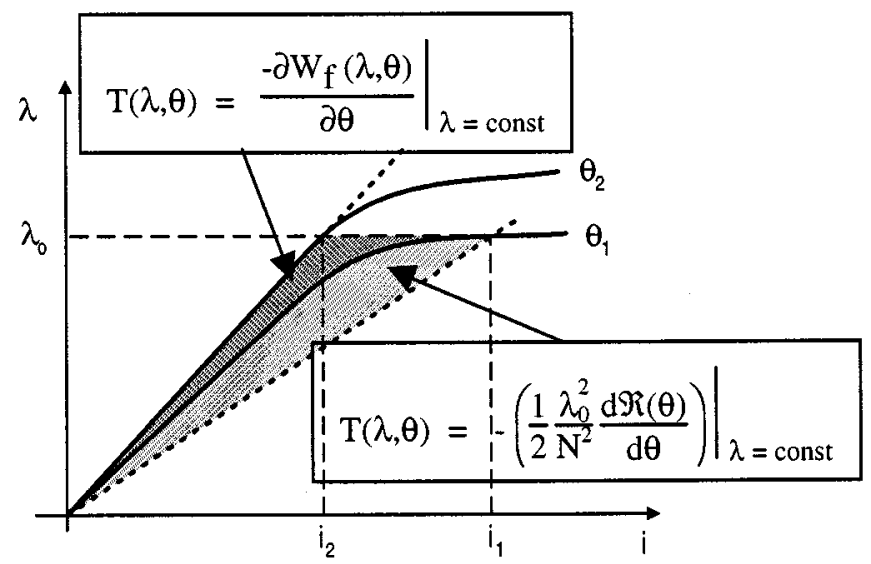

Fig. 8. Plots of flux linkage versus current showing torque as areas (divided by incremental position) calculated from the general torque equation and the unsaturated torque equation.

Their torque control strategy was to determine flux linkage as a function of current and invert this to get current as a function of flux linkage. The commanded flux linkage would be given by manipulation of (23) given in

$$
\lambda^{*}=\left(\frac{-2 N^{2} T^{*}}{\frac{d \Re}{d \theta}}\right)^{1 / 2} .
$$

Their assertion was that since the magnetic nonlinearities were modeled by the flux linkage term, then (23) was also valid for the saturated SRM. This is incorrect because the reluctance term is not just a function of position but is also a function of flux level. Fig. 8 shows graphically the actual torque produced for a saturated SRM at a given flux linkage, $\lambda_{o}$, overlaid on top of the torque obtained using the unsaturated torque equation (23). The actual torque is the area bounded by the flux linkage versus current curves for the two different positions and the horizontal line at the constant value of flux linkage, $\lambda_{o}$, in Fig. 8.

The torque obtained using the unsaturated torque equation (23) is shown as the light gray area underneath the actual torque. It is the triangular area bounded by the straight lines for the two different positions. Note that the area (and, therefore, torque) that the unsaturated equation predicts is larger than the actual area (torque) of the saturated SRM. This approach does not accurately predict the torque produced by a saturated SRM.

This approach, however, has some merit. If an appropriate operating point reluctance derivative is formed, then the flux linkage can be solved for in terms of the commanded torque. The question which must be answered is what is the appropriate operating point reluctance derivative.

Assuming an operating point equation of torque given by

$$
T_{\mathrm{op}}\left(\lambda_{o}, \theta_{\mathrm{op}}\right)=-\left(\frac{1}{2} \frac{\lambda_{0}^{2}}{N^{2}} \frac{d \Re_{\mathrm{op}}\left(\lambda_{o}, \theta_{\mathrm{op}}\right)}{d \theta}\right)
$$




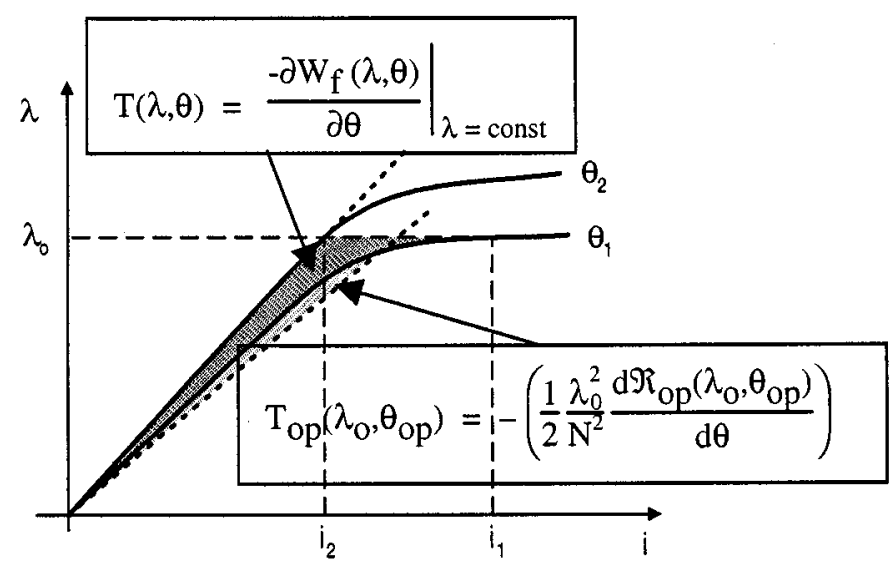

Fig. 9. Plots of flux linkage versus current showing torque as areas calculated from the general torque equation and the operating point torque equation.

an operating point reluctance derivative is given by equating the torque expressions (10) and (25). Solving for the operating point reluctance derivative gives

$$
\frac{d \Re_{\mathrm{op}}\left(\lambda_{o}, \theta_{\mathrm{op}}\right)}{d \theta}=-2 N^{2} \frac{\frac{-\partial W_{f}\left(\lambda_{o}, \theta_{\mathrm{op}}\right)}{\partial \theta}}{\lambda_{0}^{2}}
$$

Equation (26) ensures that for a given flux linkage, the area between the flux linkage versus current curves will be the same as would be obtained using (10). Since the torque over a given change in rotor position is equal to this area, (26) ensures that the torque will be correct at the given operating point $\lambda_{o}$. Fig. 9 shows the areas obtained from the general torque equation and the operating point torque equation (25) are equal at the given flux linkage $\lambda_{o}$ and for the given rotor position.

\section{Operating Point TORQue MOdel IN AN SRM USING CURRENT AND Rotor POSITION AS STATE VARIABLES}

The unsaturated torque equation in terms of phase currents and inductance derivatives is commonly expressed as in

$$
T\left(\theta_{r}\right)=\frac{1}{2} i\left(\theta_{r}\right)^{2} \frac{d L\left(\theta_{r}\right)}{d \theta_{r}}
$$

This expression for torque can be derived from (19) and (20). Assuming an unsaturated SRM, the flux linkage is no longer a function of current and can be expressed as

$$
\lambda(\theta)=L(\theta) i
$$

From (20), the co-energy is given in

$$
W_{\mathrm{co}}\left(i_{o}, \theta\right)=\int_{0}^{i_{o}} L(\theta) i d i=\frac{1}{2} i_{o}^{2} L(\theta) .
$$

Equation (27) comes directly from differentiating (29) with respect to rotor position. The form of (27) is very similar to the form of the unsaturated torque equation expressed in terms of flux linkage and the reluctance derivative (23).

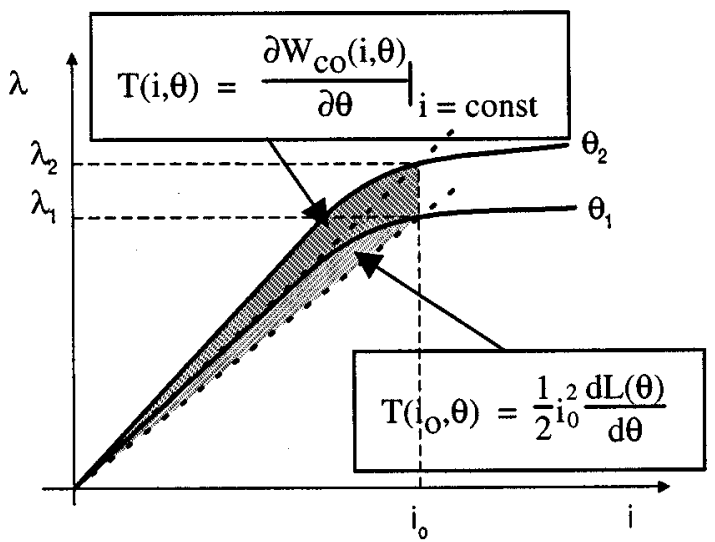

Fig. 10. Plots of flux linkage versus current showing torque as areas calculated from the general torque equation and the unsaturated torque equation.

In order to find an appropriate operating point model, the same approach as described in Section III is used. Assuming an operating point equation of torque given in

$$
T_{\mathrm{op}}\left(i_{o}, \theta_{\mathrm{op}}\right)=\frac{1}{2} i_{0}^{2} \frac{d L_{\mathrm{op}}\left(i_{o}, \theta_{\mathrm{op}}\right)}{d \theta}
$$

an operating point inductance derivative is given by equating the torque expressions (19) and (30). Solving for the operating point inductance derivative gives

$$
\frac{d L_{\mathrm{op}}\left(i_{o}, \theta_{\mathrm{op}}\right)}{d \theta}=2 \frac{\frac{\partial W_{\mathrm{co}}\left(i_{o}, \theta_{\mathrm{op}}\right)}{\partial \theta}}{i_{0}^{2}} .
$$

Equation (31) ensures that, for a given phase current and change in rotor position, the area between the flux linkage versus current curves will be the same as would be obtained using (19). Since the torque over a given change in rotor position is equal to this area, (31) ensures that the torque will be correct at the given operating point $i_{o}$.

Fig. 10 shows graphically the actual torque produced for a saturated SRM at a given current $i_{o}$ overlaid on top of the torque obtained using the unsaturated torque equation (27).

The actual torque is the area bounded by the flux linkage versus current curves for the two different positions and the vertical line at the constant value of current $i_{o}$. The torque obtained using the unsaturated torque equation (27) is shown as the light gray area underneath the actual torque. It is the triangular area bounded by the straight lines for the two different positions. Note that the area (and, therefore, torque) that the unsaturated equation produces is smaller than the actual area (torque) of the saturated SRM because the unsaturated model approximates the area with straight lines from the origin to the given operating point flux linkage.

Equation (31) predicts the correct inductance derivative in order to produce the same average torque that the general torque equation (19) predicts. Fig. 11 shows the areas obtained from the general torque equation and the operating point torque equation (31) are equal at the given current $i_{o}$ and for the given rotor position. 


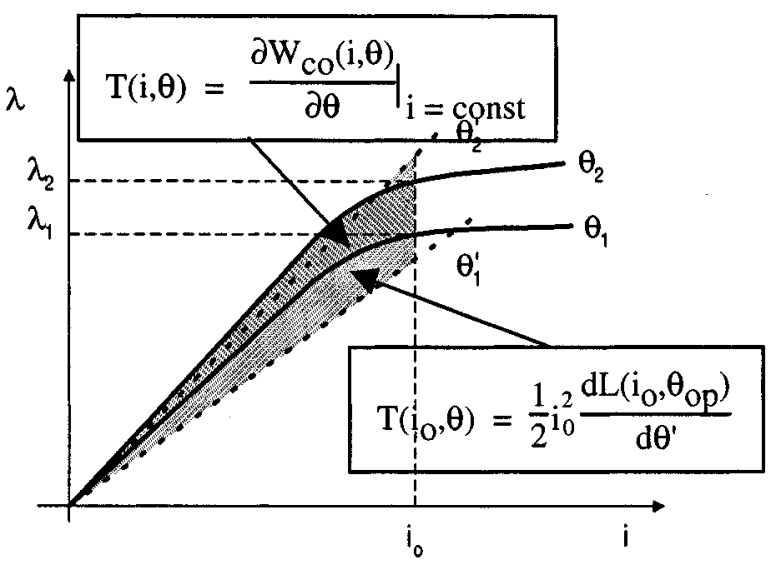

Fig. 11. Plots of flux linkage versus current showing torque as areas calculated from the general torque equation and the operating point torque equation.

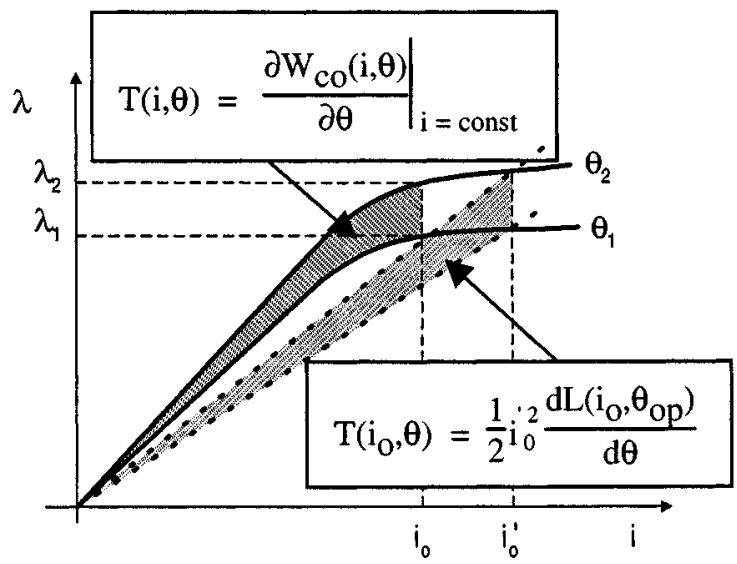

Fig. 12. Plots of flux linkage versus current showing torque as areas calculated from the general torque equation and the operating point torque equation using the same change in position.

However, the change in rotor position has increased for the same current level in order to achieve the same area, and is shown as $\theta_{1}^{\prime}$ and $\theta_{2}^{\prime}$. The area is equal to the mechanical energy. Since the mechanical energy is the product of the average torque and the change in position, and the torque decreases as the SRM saturates, the change in position has to increase in order for the product to remain constant.

An alternative way to view the operating point inductance derivative is to constrain the change in position to be constant, shown graphically in Fig. 12.

The current using the operating point model, $i_{o}^{\prime}$, is larger than the current used by the general model in order to produce a given amount of torque. Fig. 12 shows that the commanded current must be increased from its nominal value for a commanded torque when using the operating point torque equation (30).

The current must be increased for a commanded value of torque because of the operating point equation. The form of the operating point equation is the same as the unsaturated torque equation. As the machine saturates, the torque per ampere (or torque per ampere squared, as it is commonly described) decreases. To compensate for this, the current must be increased.

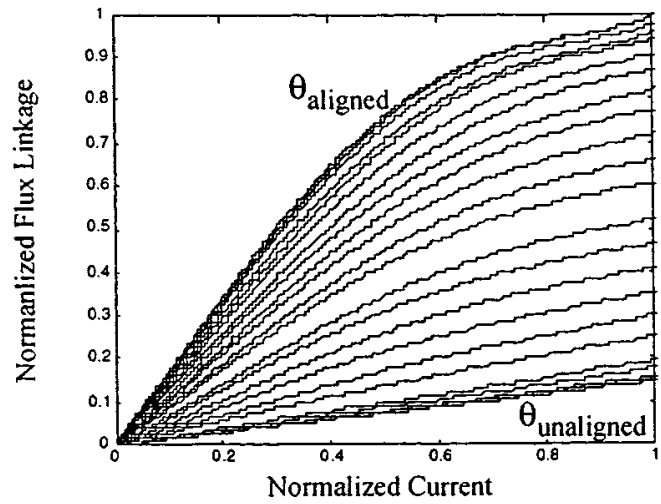

Fig. 13. Normalized flux linkage versus current at various rotor positions for a typical SRM.

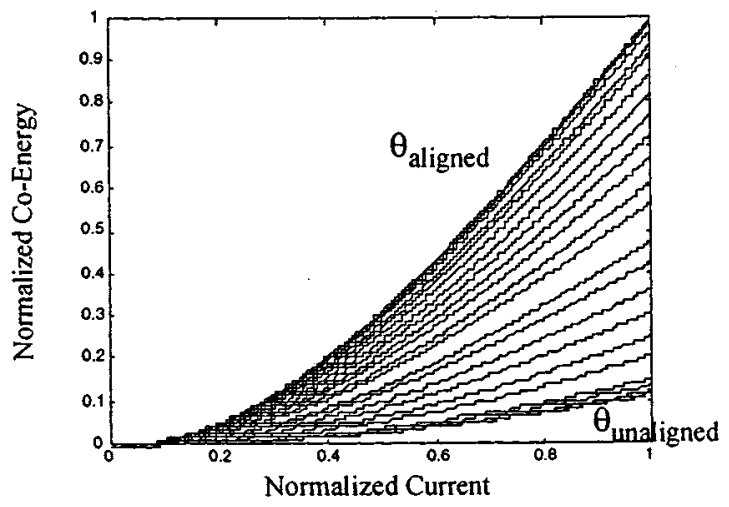

Fig. 14. Normalized co-energy versus current at various rotor positions for a typical SRM.

\section{MOdELING OF A SATURATED SMR}

When the SRM is not saturated, the flux linkage in a given phase varies linearly with current in the given phase. The slope of this linear variation is the inductance. As the current is increased in a given phase, the iron begins to saturate and the flux linkage no longer varies linearly with current. Fig. 13 shows a typical normalized flux linkage versus current curve for an SRM.

In order to accurately model the saturated SRM, the torque has to be expressed as a nonlinear function of flux linkage or current and rotor position. This is accomplished by use of the flux linkage versus current curves at different rotor positions as shown in Fig. 13. Using current and position as the state variables, the torque is evaluated in terms of the general torque equation (19) and the co-energy is evaluated by (20).

The flux linkage versus current curves are either measured or calculated using a finite-element method analysis. Once the flux linkage versus current curves are obtained, a functional representation of co-energy must be derived. There are several methods to approximate the co-energy as a function of current and position. It is beyond the scope of this paper to evaluate each of these methods. The simplest method, however, is to curve fit the flux linkage versus current for a given rotor position. Then, a curve is fitted to each of these flux linkage versus current curves with respect to rotor position in order to get the co-energy as a 


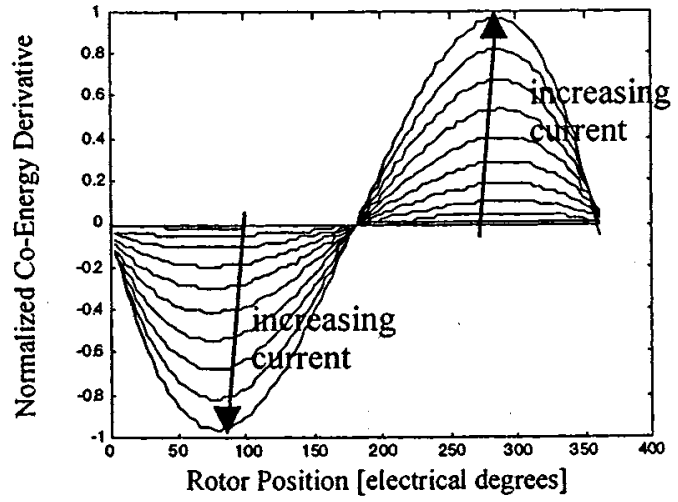

Fig. 15. Normalized spatial derivative of co-energy versus rotor positions at various currents for a typical SRM.

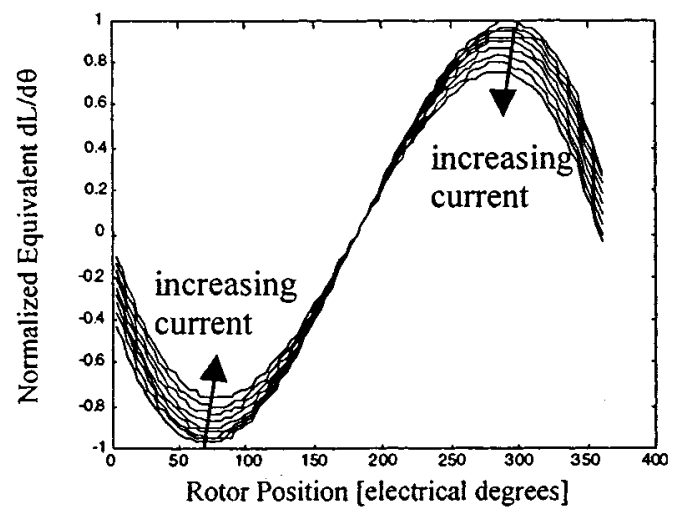

Fig. 16. Normalized equivalent spatial inductance derivative versus rotor positions at various currents for a typical SRM.

function of two variables, current and position. Fig. 14 shows normalized plots of co-energy versus current at various rotor positions. These curves represent the integration of the flux linkage versus current function with respect to current (20).

This co-energy function is then differentiated in order to get a representation for torque as a function of current and position using (19). Fig. 15 shows normalized plots of the spatial derivative of co-energy (which is equal to torque) versus rotor position at various current levels. The aligned position of the rotor and stator poles for the given phase corresponds with zero electrical degrees in Fig. 15. As the rotor position increases, the torque is negative because the reluctance force tends to pull the rotor back toward alignment.

As the electrical angle increases past $180^{\circ}$, the torque is positive because the reluctance force tends to pull the next rotor pole toward alignment in the direction of rotation.

Fig. 16 shows normalized plots of the operating point (or equivalent) spatial inductance derivative versus position at various current levels. The operating point inductance derivative decreases with current level because the effective torque per ampere (or torque per ampere squared) decreases with saturation. The amplitude of this equivalent inductance derivative is a function of position and current.
TABLE I

NOMINAL PARAMETERS OF OULTON 10-hp SRM

\begin{tabular}{ll}
\hline Parameter & Value \\
\hline model number & PLD 132 \\
$\mathrm{r}_{\mathrm{s}}$ & $0.8 \Omega$ \\
$\mathrm{L}_{\mathrm{s}}$ (aligned) & $268 \mathrm{mH}$ \\
$\mathrm{L}_{\mathrm{s}}$ (unaligned) & $24 \mathrm{mH}$ \\
rated current & $16.0 \mathrm{amp}$ \\
rated voltage & 380 volt d.c. bus \\
stator /rotor poles & $8 / 6$ \\
phases & 4 \\
\end{tabular}

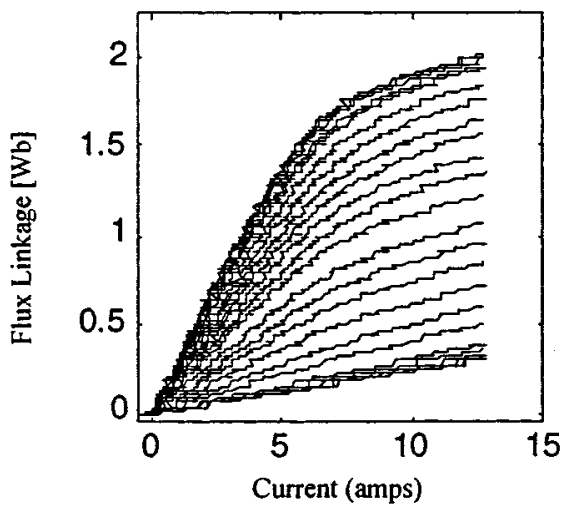

Fig. 17. Measured flux linkage versus current for one phase of the Oulton SRM for one electrical revolution (13-A commanded current).

\section{EXPERIMENTAL RESULTS}

The operating point inductance derivative of Section $\mathrm{V}$ was used with the control strategy discussed in [7] and [12]. This control strategy with the operating point model was tested on a 10-hp Oulton SRM, the parameters of which are shown in Table I. Fig. 17 shows the measured flux linkage versus current plot for the Oulton SRM, from which it can be seen that this machine starts to saturate at approximately $5 \mathrm{~A}$.

Fig. 18 shows the measured and curve-fit operating point inductance derivative curves for the SRM. Curve-fitted unsaturated inductance derivative curves are superimposed in Fig. 18 to illustrate the reduction in the equivalent (operating point) inductance derivative with current. This is an effective reduction in torque per ampere when the machine saturates.

Fig. 19 shows plots of the torque signal for the Oulton SRM. The top trace is the torque produced by the SRM with square-wave excitation. The middle trace shows the torque produced using the rotating vector methods described in [7] and [12] using the measured unsaturated inductance derivative values. The bottom trace uses the iterative rotating vector methods described in [7]. This method uses the operating point inductance derivative in order to calculated the currents necessary to reduce torque ripple in the saturated SRM.

It is clear from Fig. 19 that the torque ripple is significantly reduced using the operating point inductance derivative described in this paper. 

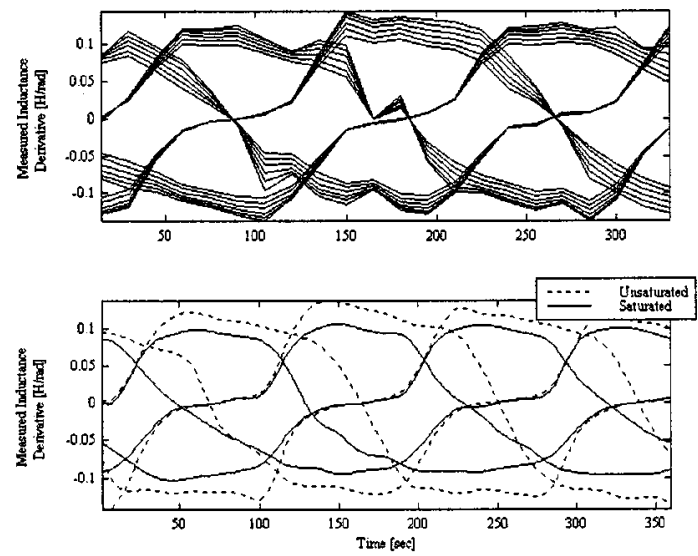

Fig. 18. Measured operating point inductance derivatives for the saturated Oulton SRM (top trace), and curve-fit inductance derivatives (bottom trace).
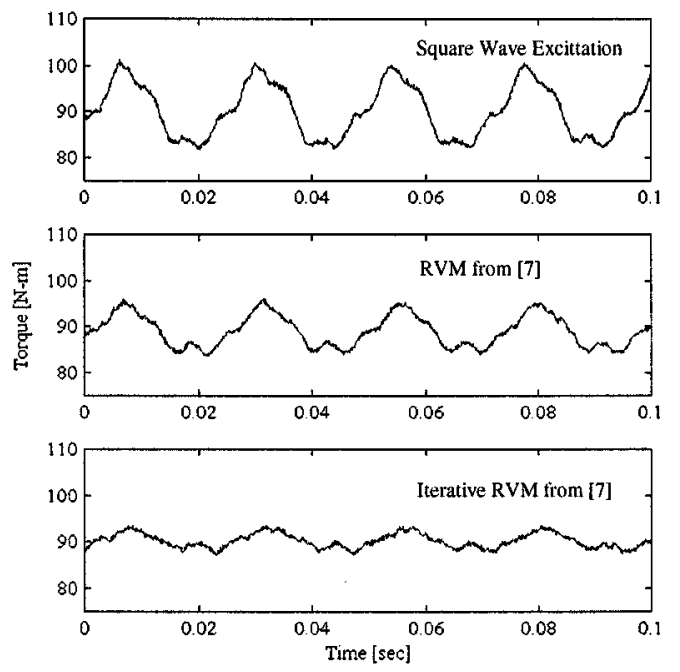

Fig. 19. Measured torque for the Oulton SRM using square-wave excitation (top trace), currents derived from the unsaturated RVM [7], [12] (middle trace), and currents derived from the iterative RVM [7] (bottom trace).

\section{CONCLUSION}

This paper has presented an analysis of torque production in SRM's. Torque was defined in two ways: 1) in terms of rotor position and current and 2) in terms of rotor position and flux linkage. A graphical interpretation was provided for both forms.

A correct operating point torque expression for a saturated SRM was derived based on the unsaturated SRM for both cases. A graphical interpretation was provided to show correct calculation methods and potential errors.

The operating point method was applied to a highly saturated commercial SRM to validate the correctness of the methods presented here.
This analysis allows SRM torque control strategies based on the unsaturated torque equation to be implemented on a saturated machine via a correct operating point model.

\section{REFERENCES}

[1] K. Russa, I. Husain, and M. Elbuluk, "Torque ripple minimization in switched reluctance machines over a wide speed range," in Conf. Rec. IEEE-IAS Annu. Meeting, New Orleans, LA, Oct. 5-9, 1997, pp. 668-675.

[2] A. M. Stankovic, G. Tadmor, and Z. Coric, "Low torque ripple control of current-fed switched reluctance motors," in Conf. Rec. IEEE-IAS Annu. Meeting, Oct. 1996, pp. 84-91.

[3] M. O. Bilgic, V. Ozbulur, and A. Sabanovic, "Torque ripple minimization of a switched reluctance motor," in Proc. IEEE APEC'95, 1995, pp. 406-410.

[4] P. C. Kjaer, J. J. Gribble, and T. J. E. Miller, "High-grade control of switched reluctance machines," in Conf. Rec. IEEE-IAS Annu. Meeting, San Diego, CA, Oct. 1996, pp. 92-100.

[5] R. S. Wallace and D. G. Taylor, "A balanced commutator for switched reluctance motors to reduce torque ripple," IEEE Trans. Power Electron., vol. 7, pp. 617-626, Oct. 1992.

[6] I. Husain and M. Ehsani, "Torque ripple minimization in switched reluctance motor drives by PWM current control," IEEE Trans. Power Electron., vol. 11, pp. 83-88, Jan. 1996.

[7] N. J. Nagel and R. D. Lorenz, "Complex rotating vector methods for smooth torque control of a saturated switched reluctance motor," in Conf. Rec. IEEE-IAS Annu. Meeting, vol. 4, Phoenix, AZ, Oct. 1999, pp. 2591-2598.

[8] B. Y. Ma, T. H. Liu, and W. S. Feng, "Modeling and torque pulsation reduction for a switched reluctance motor drive system," in Proc. IEEE Int. Conf. Industrial Electronics, Los Alimos, CA, June 1996, pp. 72-77.

[9] J. W. Finch, H. M. B. Metwally, and J. A. Agber, "Performance prediction in saturated variable reluctance and hybrid motors," in IEEE Int. Conf. Power Electronics, New Delhi, India, 1996, pp. 231-236.

[10] T. J. E. Miller, Switched Reluctance Motors and Their Control, Oxford, U.K.: Clarendon, 1993.

[11] N. L. Schmitz and D. W. Novotny, Introductory Electromechanics. New York: Ronald, 1965.

[12] N. J. Nagel and R. D. Lorenz, "Rotating vector methods for sensorless, smooth torque control of a switched reluctance motor drive," in Conf. Rec. IEEE-IAS Annu. Meeting, vol. 1, St. Louis, MO, Oct. 1998, pp. 723-730.

[13] N. J. Nagel, "Complex rotating vector analysis and control of a switched reluctance motor," Ph.D. dissertation, Dep. Mech. Eng., University of Wisconsin, Madison, WI, Dec. 1998.

[14] F. Filicori, C. G. Lo Bianco, and A. Tonielli, "Modeling and control strategies for a variable reluctance direct-drive motor," IEEE Trans. Ind. Electron., vol. 40, pp. 105-114, Feb. 1993.

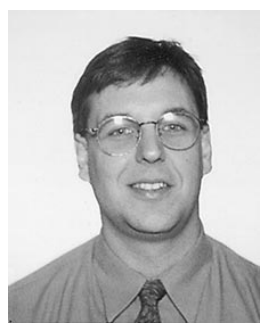

Nicholas J. Nagel (S'95-A'98) received the B.S. degree in mechanical engineering from the University of Illinois, Champaign/Urbana, and the M.S. degrees in mechanical engineering and electrical and computer engineering and the Ph.D. degree in mechanical engineering from the University of Wisconsin, Madison, in 1989, 1995, 1996, and 1998, respectively, with a focus in control of electric machinery.

$\mathrm{He}$ is currently with MPC Products Corporation, Skokie, IL, where his research is focused on highperformance motion and motor control for aerospace applications. His interests include electric machines, machine drives, control systems, power electronics, and electromechanics. 


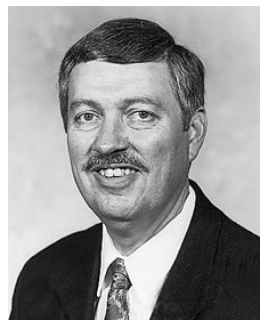

Robert D. Lorenz (S'83-M'84-SM'91-F'98) received the B.S., M.S., and Ph.D. degrees from the University of Wisconsin, Madison, in 1969, 1970, and 1984 , respectively.

Since 1984, he has been a member of the faculty of the University of Wisconsin, Madison, where he is the Consolidated Papers Foundation Professor of Controls Engineering in both the Mechanical Engineering and Electrical and Computer Engineering Departments. In this position, he acts as Codirector of the Wisconsin Electric Machines and Power Electronics Consortium. He is also an active Consultant to many organizations. He was a Visiting Research Professor in the Electrical Drives Group, Catholic University of Leuven, Leuven, Belgium, in the summer of 1989 and at the Power Electronics and Electrical Drives Institute, Technical University of Aachen, Aachen, Germany, in the summers of 1987, 1991, 1995, 1997, and 1999. In 1969-1970, he did the Master thesis research at the Technical University of Aachen. From 1972 to 1982, he was a member of the research staff at the Gleason Works, Rochester, NY. His current research interests include sensorless electromagnetic motor/actuator technologies, real-time signal processing and estimation techniques, precision multiaxis motion control, and ac drive and high-precision machine control technologies.

Dr. Lorenz is currently the Vice President/President Elect of the IEEE Industry Applications Society (IAS), a Distinguished Lecturer of the IAS for 2000/2001, the immediate past Chair of the IAS Awards Department, past Chairman of the IAS Industrial Drives Committee, and a member of the IAS Industrial Drives, Electric Machines, Industrial Power Converter, and Industrial Automation and Control Committees. He is a member of the IEEE Sensor Council AdCom and the IEEE Neural Network AdCom. He is a Registered Professional Engineer in the States of New York and Wisconsin. He is also a member of the American Society of Mechanical Engineers, Instrument Society of America, and Society of Photo-Optical Instrumentation Engineers. 